\title{
Comparison of Different Targeting Methods for Subthalamic Nucleus Deep Brain Stimulation
}

\author{
Ting Guo ${ }^{1,3}$, Kirk W. Finnis ${ }^{4}$, Sean C.L. Deoni ${ }^{5}$, \\ Andrew G. Parrent ${ }^{2}$, and Terry M. Peters ${ }^{1,3}$ \\ ${ }^{1}$ Robarts Research Institute \\ ${ }^{2}$ The London Health Sciences Centre, London, ON, Canada N6A 5K8 \\ ${ }^{3}$ Biomedical Engineering, University of Western Ontario, London, ON, Canada N6A 5B9 \\ ${ }^{4}$ Atamai Inc, London, Ontario, Canada N6B 2R4 \\ ${ }^{5}$ Centre for Neuroimaging Sciences, Institute of Psychiatry, King's College, London, UK \\ \{tguo, tpeters\} @imaging.robarts.ca
}

\begin{abstract}
The subthalamic nucleus (STN) has been adopted as a commonly used surgical target in deep brain stimulation (DBS) procedures for the treatment of Parkinson's disease. Many techniques have been developed to facilitate STN DBS targeting, and consequently to improve the surgical outcome. In this work, we conducted a retrospective study on 10 patients who were treated with bilateral STN DBS to assess the target localization accuracy and precision of six methods in STN DBS surgery. A visualization and navigation system integrated with normalized functional and anatomical information was employed to perform the targeting procedures. Actual surgical target location determined by an experienced neurosurgeon with pre-operative image-guided surgical target/trajectory planning and intra-operative electrophysiological exploration and confirmation was considered as the "gold standard" in this evaluation and was compared with those localized using each of the six targeting methods. The mean distance between the actual surgical targets and those planned was $3.0 \pm 1.3 \mathrm{~mm}, 3.2 \pm 1.1 \mathrm{~mm}, 2.9 \pm 1.1 \mathrm{~mm}, 2.7 \pm$ $1.2 \mathrm{~mm}, 2.5 \pm 1.0 \mathrm{~mm}$, and $1.7 \pm 0.8 \mathrm{~mm}$ for targeting approaches based on $\mathrm{T}_{2^{-}}$ weighted magnetic resonance image (MRI), brain atlas, $\mathrm{T}_{1}$ and $\mathrm{T}_{2}$ maps, electrophysiological database, collection of final surgical targets of previous patients, and the combination of these functional and anatomical data respectively. The results demonstrated that the use of functional data along with anatomical data provides reliable and accurate target position for STN DBS.
\end{abstract}

\section{Introduction}

The subthalamic nucleus (STN), which is an obliquely oriented biconvex lens-shaped mass of grey matter, located dorsal to the internal capsule rostral to the substantia nigra within the caudal diencephalon in the ventral part of the subthalamus, plays an important role in the basal ganglia-thalamocortical motor circuit. Considered as the main "driving" factor in basal ganglia, the STN utilizes the excitatory neurotransmitter glutamate and innervates both basal ganglia output nuclei, the internal segment of the globus pallidus (GPi) and the pars reticulata of the substantia 
nigra $(\mathrm{SNr})$. The generation of the abnormal patterns of activity observed in Parkinson's disease and essential tremor is thought to be caused by the hyperactivity of the STN. Therefore, interruption of STN activity by creating a small lesion or using high frequency deep brain stimulation (DBS) alleviates motor abnormalities such as tremor, rigidity, bradykinesia, postural instability, and akinesia [1].

Since the STN is surrounded by delicate deep-brain structures and its average size is very small, i.e. $5.9 \mathrm{~mm}, 3.7 \mathrm{~mm}$, and $5 \mathrm{~mm}$ in anteroposterior, mediolateral, and dorsoventral dimensions respectively [2], it is critical to localize the surgical target accurately with minimal invasiveness to achieve the optimal surgical outcome. In clinical practice, prior to the final lesion creation or stimulator placement, the surgical targeting is currently primarily determined by pre-operative image-guided surgical target/trajectory planning and intra-operative electrophysiological confirmation. The first phase is carried out based on pre-operative magnetic resonance (MR) or computed tomography (CT) images, whereas the second step involves invasive intraoperative electrophysiological measurements to refine the optimal surgical targets.

To enhance the targeting accuracy and reduce the invasiveness, clinicians have employed a variety of techniques including printed and digitized anatomical brain atlases [3,4], $\mathrm{T}_{2}$-weighted $\mathrm{MR}$ images [5], high resolution $\mathrm{T}_{1}$ and $\mathrm{T}_{2}$ maps [6], electrophysiological databases [7], registered surgical targets of previous patients [8], as well as integration of multiple functional and anatomical references [9]. Anatomical atlases contain information about subsections of deep-brain nuclei, which are usually not distinguishable on regular MR images. However the atlases derived from single cadaver brain specimen or multiple subject brain hemispheres may not be able to provide sufficient statistical representation of the population and be difficult to generalize. Special MR $\mathrm{T}_{1}$ and $\mathrm{T}_{2}$ maps with excellent resolution and signal to noise ratio carry the disadvantage of lack of anatomical variation. Patient specific $\mathrm{T}_{2}$ weighted MR images may allow better visualization of the STN, nevertheless the inconsistency of the target positions defined with $\mathrm{T}_{2}$-weighted $\mathrm{MR}$ images and with electrophysiological explorations should be used with caution [10,11].

Stereotactic targeting of the STN using registered surgical targets and electrophysiological databases, which contains data from multiple patients normalized into a standard brain template, is more promising, because they provide valuable probabilistic maps of population-based actual target information and deep-brain electrophysiological activity. Furthermore, the integration of multiple functional and anatomical references can establish the relationship between functional organization and anatomic structures for estimating the surgical targets. In this work, we conducted a retrospective study to evaluate and compare the target localization accuracy and precision of six targeting methods in STN DBS surgery.

\section{Materials and Methods}

\subsection{Patients and MRI Data}

Patient selection: MR images of 10 patients, who were treated with bilateral STN DBS procedures to alleviate their Parkinson's disease symptoms at London Health Sciences Centre (LHSC), London, Ontario, Canada, were selected from the database 
containing data of 26 (15 male and 11 female) patients (21-79 years, mean 59.7 years), who had undergone a total of 49 STN DBS procedures at LHSC.

MRI Data: We acquired pre-operative $\mathrm{T}_{1}$-weighted MR images using a 3D SPGR sequence, and $\mathrm{T}_{2}$-weighted $\mathrm{MR}$ images using a $2 \mathrm{D}$ fast spin-echo sequence on a $1.5 \mathrm{~T}$ GE Signa scanner with the imaging parameters listed in Table 1.

Table 1. Imaging parameters of $\mathrm{T}_{1}$-weighted and $\mathrm{T}_{2}$-weighted $\mathrm{MR}$ images

\begin{tabular}{|c|c|c|c|c|c|c|}
\hline $\begin{array}{c}\text { Imaging } \\
\text { Modality }\end{array}$ & $\begin{array}{c}\text { TR } \\
(\mathbf{m s})\end{array}$ & $\begin{array}{c}\text { TE } \\
(\mathbf{m s})\end{array}$ & $\begin{array}{c}\text { Flip } \\
\text { Angle }\end{array}$ & NEX & Voxel Size & Dimension \\
\hline $\mathrm{T}_{1}$-weighted & 8.9 & 1.9 & $20^{\circ}$ & 2 & $1.17 \times 1.17 \times 1 \mathrm{~mm}^{3}$ & $256 \times 256 \times 248$ \\
\hline $\mathrm{T}_{2}$-weighted & 2800 & 110 & $90^{\circ}$ & 4 & $1.0 \times 1.0 \times 1.5 \mathrm{~mm}^{3}$ & $256 \times 256 \times 21$ (coronal) \\
\cline { 6 - 7 } & & & & & & \\
\hline
\end{tabular}

\section{2 “Gold Standard” and Surgical Procedure}

Gold standard: Actual surgical target locations determined by an experienced neurosurgeon using standard surgical procedures were considered as the "gold standard" in this evaluation and were compared with those localized using each of the six targeting methods.

Surgical procedure: The Leksell G stereotactic frame (Elekta Instruments AB, Stockholm, Sweden) with attached MR compatible localizer was applied to each patient immediately before imaging. After image acquisition, the neurosurgeon would identify the internal landmarks, anterior commissure (AC) and posterior commissure (PC), relative to the frame system on the MR images, reconstruct the images in ACPC-based coordinates, align the nearest section of an anatomical brain atlas to the images, and select the initial the surgical target. Before the final placement of the deep brain stimulator, five electrodes were applied to obtain the micro-recording and macro-stimulation measurements on a track extending $10 \mathrm{~mm}$ above and below the initially planned target and to eventually localize the optimal surgical position.

\subsection{Visualization and Navigation System}

A comprehensive visualization and navigation system integrated with normalized functional and anatomical information [9] within the standard brain space was employed to perform the surgical targeting procedures in our study. This system incorporates a digitized and segmented anatomical brain atlas [3], a 3D electrophysiological database, high resolution $\mathrm{T}_{1}$ and $\mathrm{T}_{2}$ maps [6], and collections of real surgical targets. It is also capable of interactively displaying the linked patient image and standard brain template, reformatting them along arbitrary axes, and registering and fusing images from different modalities, digitized brain atlases, and functional data with the pre-operative images. The system readily accommodates inter-subject anatomical variability through the use of a fast three-dimensional nonrigid image registration approach, AtamaiWarp [12]. Virtual surgical probes may also be manipulated within this environment to allow data-sampling points to be readily 
visualized with respect to pre-defined targets and previously electrophysiological data imported from the database.

\subsection{Targeting Techniques}

At our institution, the dorsolateral portion of STN is regarded as the most effective stimulation site in STN DBS. Therefore, all the target positions estimated using the following targeting techniques were selected based on this principle.

a) $T_{2}$-weighted MRI-based targeting: Because of the presence of a high concentration of iron within the STN, which is located lateral to the red nucleus and dorsolateral to the substantia nigra, the anterior part of the nucleus has hypointense signal intensity on $\mathrm{T}_{2}$-weighted $\mathrm{MR}$ images. Although this signal is not always present in the posterior part of the nucleus, it is visible in the anterior portion and correlates well with the red nucleus and substantia nigra to assist the identification of the surgical target location within the STN. The $\mathrm{T}_{2}$-weighted coronal and axial acquisitions of each patient are fused with his/her pre-operative $\mathrm{T}_{1}$-weighted image. The target coordinates were defined at a point dorsolateral to the centre of the STN on the three intersecting slices of these overlapped images.

b) Anatomical brain atlas-based targeting: The digitized version of the wellestablished Schaltenbrand and Wahren stereotactic brain atlas [3] and the segmented STN derived from this atlas were non-rigidly transformed to each patient brain image space using the AtamaiWarp algorithm. A colour-coded atlas, combined with a meshrepresentation of the segmented nucleus and the automatically computed STN centroid, permits intuitive planning of the surgical trajectory inside of the nucleus.

Table 2. Imaging parameters of DESPOT1 and DESPOT2

\begin{tabular}{|c|c|c|c|c|c|c|}
\hline $\begin{array}{c}\text { Imaging } \\
\text { Modality }\end{array}$ & $\begin{array}{c}\text { TR } \\
(\mathbf{m s})\end{array}$ & $\begin{array}{c}\text { TE } \\
(\mathbf{m s})\end{array}$ & $\begin{array}{c}\text { Flip } \\
\text { Angle }\end{array}$ & $\begin{array}{c}\text { BW } \\
(\mathbf{k H z})\end{array}$ & Voxel Size & Sequence \\
\hline DESPOT1 & 11.4 & 2.9 & $4^{\circ}, 16^{\circ}$ & \pm 7.81 & $0.34 \mathrm{~mm}^{3}$ & SPGR \\
\hline DESPOT2 & 4.2 & 2.1 & $15^{\circ}, 55^{\circ}$ & \pm 62.5 & $0.34 \mathrm{~mm}^{3}$ & SSFP \\
\hline
\end{tabular}

c) $T_{1}$ and $T_{2}$ map-based targeting: To provide a noninvasive quantitative imaging component to our system, high resolution $\left(0.34 \mathrm{~mm}^{3}\right.$ isotropic) and signal to noise ratio $T_{1}$ and $T_{2}$ maps of the deep brain region of a young healthy male volunteer were generated by mutually co-registering and averaging $55 \mathrm{~T}_{1}$ maps and $25 \mathrm{~T}_{2}$ maps acquired using the DESPOT1 and DESPOT2 methods respectively [6]. Differences among some deep-brain nuclei that appear indistinct on regular MR images are visible on the two maps. The STN on these maps was segmented using a genetic algorithm (GA) framework and incorporating characteristics of the $k$-means clustering algorithm [6]. Our surgical target identification methodology using the $\mathrm{T}_{1}$ and $\mathrm{T}_{2}$ maps is similar to that employed when using the anatomical brain atlas.

d) Electrophysiological database-based targeting: Our electrophysiological database for STN DBS contains intra-operative micro-recording and macrostimulation data acquired from 49 procedures in standard brain space. The data coded with respect to STN-specific firing patterns were retrieved from the database and 
non-rigidly mapped to each patient's MR image to approximately delineate the patient specific nucleus. The location that, when stimulated, resulted in the optimum control of the patient's symptoms was used to define the surgical target.

e) Targeting based on Actual surgical target collection: We non-rigidly registered the collection of final surgical target locations of these 49 STN DBS procedures from the standard brain coordinates to the native brain space of each patient. The automatically generated center of mass (COM) of this cluster of actual targets was directly employed as the target estimation in this targeting protocol.

f) Comprehensive functional and anatomical information-based targeting: With our visualization and navigation system, we localized the surgical targets of these 20 procedures utilizing all the customized functional and anatomical data. The combination of the brain atlas, $\mathrm{T}_{1}$ and $\mathrm{T}_{2}$ maps, neurophysiological information, and real surgical data, helped us establish the correlation between the deep-brain anatomical structures and the electrophysiological organization.

\section{Results}

We computed the mean distances between the actual surgical targets, determined by a stereotactic neurosurgeon using pre-operative MR image-based planning and intraoperative electrophysiological explorations, with those, planned by a nonneurosurgeon using six targeting techniques described above. The results are shown in table 3 below. A one-way analysis of variance (ANOVA) test analyzing the differences on surgical targeting accuracy among the six target localization methods revealed significant differences between groups $(\mathrm{F}=4.48, \mathrm{df}=5, \mathrm{p}=0.001)$. The Tukey's HSD Post-Hoc test also demonstrated that the localization accuracy of comprehensive targeting approach is significantly better than that of the methods based on $\mathrm{T}_{1}$ and $\mathrm{T}_{2}$ maps, brain atlas and $\mathrm{T}_{2}$-weighted MRI $(\mathrm{p}<0.01)$. Although no significant difference between the comprehensive approach and the method based on either the electrophysiological database $(p=0.07)$ or the collection of previous final surgical targets $(\mathrm{p}=0.25)$, the mean error of the comprehensive targeting approach is still approximately $0.9 \mathrm{~mm}$ less than that of the two methods. Figure 1 shows that the hypointense signal of STN on the patient $\mathrm{T}_{2}$-weighted image has better correlation with the segmented nucleus derived from the $T_{1}$ and $T_{2}$ maps than with that from digitized Schaltenbrand atlas. Figure 2 displays the spatial relationship between the actual surgical target of a patient and those identified with the six targeting methods.

Table 3. Absolute differences between the real surgical targets and the targets estimated with each targeting method

\begin{tabular}{|l|c|c|c|c|}
\hline & Mean $(\mathrm{mm})$ & Max $(\mathrm{mm})$ & Min $(\mathrm{mm})$ & Sd $(\mathrm{mm})$ \\
\hline $\mathrm{T}_{2}$-weighted MRI & 3.0 & 5.5 & 2.0 & 1.3 \\
\hline Brain atlas & 3.2 & 6.2 & 2.1 & 1.1 \\
\hline $\mathrm{T}_{1}$ and $\mathrm{T}_{2}$ maps & 2.9 & 5.7 & 2.2 & 1.1 \\
\hline EP database & 2.7 & 5.8 & 1.8 & 1.2 \\
\hline Previous targets & 2.5 & 4.2 & 1.4 & 1.0 \\
\hline Combination & 1.7 & 3.1 & 0.8 & 0.8 \\
\hline
\end{tabular}




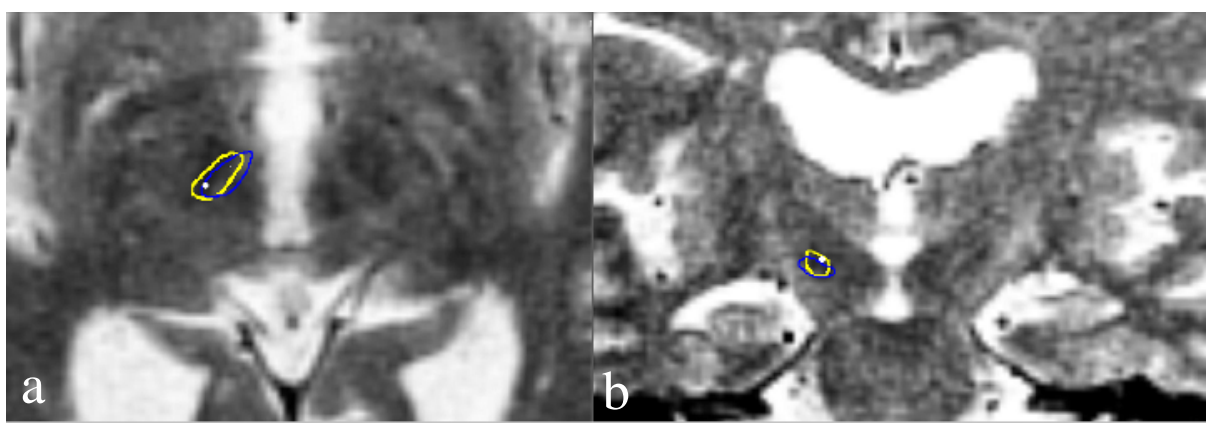

Fig. 1. $T_{2}$-weighted MR image of a patient demonstrating direct visualization of STN (a. axial slice, b. coronal slice); Yellow circle: the contour of segmented STN based on $\mathrm{T}_{1}$ and $\mathrm{T}_{2}$ maps; Blue circle: based on digitized Schaltenbrand atlas; White sphere: the actual surgical target. The digitized atlas and $\mathrm{T}_{1}$ and $\mathrm{T}_{2}$ maps were registered to the patient brain image space.

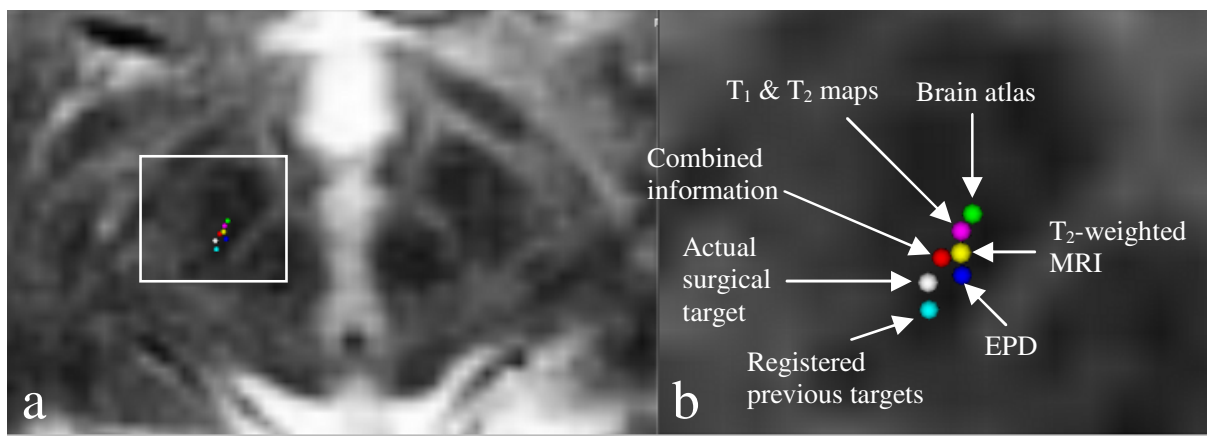

Fig. 2. Positions of surgical targets estimated with each targeting approach. Yellow sphere: target planned based on T2-weighted MR image; Green sphere: based on Schaltenbrand atlas; Magenta sphere: based on T1 and T2 maps; Blue sphere: based on electrophysiological database; Cyan sphere: based on previous surgical targets; Red sphere: based on combination of functional and anatomical data; White sphere: "gold standard" actual surgical target.

In summary, the electrophysiological database, actual surgical target collection, and the integration of approaches based on functional and anatomical information, provided more accurate initial estimation of the surgical target positions than those techniques dependent solely on anatomical references. The location of the surgical target estimated with the comprehensive functional and anatomical information-based targeting method fall within $2 \mathrm{~mm}$ of the optimal surgical target, indicating the importance of correlating functional organization with deep-brain anatomy. Practically, the current spread generated at the commonly administered stimulation levels is in the range of $2 \mathrm{~mm}$ [14], hence the optimal surgical target region may be easily approached by slightly adjusting the electrode placed at this technique planned initial position. The standard deviation of the combined method was also smaller than that of the other five methods, demonstrating that the targeting precision of this 
technique was superior to others. The integration of different functional and anatomical information, contained within the neurosurgical visualization and navigation system, can compensate for their inherent shortcomings and take advantage of their respective strengths to effectively localize the surgical target for STN DBS procedures. Since non-rigid registration was involved in most of the target localization processes, possible errors may partially come from the registration algorithm, which has a mean registration error of $1.0 \pm 0.7 \mathrm{~mm}[13]$.

\section{Discussion}

In this work, we conducted a retrospective study on 10 patients who were treated with bilateral STN DBS (20 procedures in total) to evaluate the target localization accuracy and precision of six targeting methods in STN DBS surgery. The data of these 10 patients were selected from the database containing data of 26 patients undergone a total of 49 STN DBS procedures at LHSC. The results demonstrated that each method was effective, but the target location estimated with the comprehensive functional and anatomical information-base targeting method was much closer to the optimal surgical target position than that planned using other five approaches. Also, the reduced standard deviation using this targeting method helps ensure precision of localizing the optimal surgical target. Since each of the five methods provides different unique anatomical or functional information, the combination of these references can complement the information provided by the individual methods. Although in some cases the surgical targets defined using the combination of two or three methods are close to those determined by the combination of five, the results vary from case to case and the optimal estimation of the surgical target can not be guaranteed. Using the average position of a repeatedly localized target may reduce the variance and improve the precision of targeting with each method. This can also be achieved by utilizing the comprehensive multiple functional and anatomical references. Because the functional activity does not always exactly correspond to the anatomy, establishing the correlation between the customized population-based electrophysiological database and the patient-specific detailed anatomical structures is necessary to improve the accuracy and precision of targeting. Although accurate surgical target initiation may reduce the need for invasive intra-operative exploration, and thereby decrease the surgical duration and procedure-related complications, certain intra-operative electrophysiological measurements may still be required to compensate possible inadequacy of these targeting methods. During the operation, brain tissues may shift slightly from the pre-operative MR image due to the cranial opening, the electrode insertion, and the cerebrospinal fluid (CSF) leaking. Ideally, one would characterize the degree of brain shift by registering the intra-operative MR image to the pre-operative image. However, due to the unavailability of an intraoperative MR image, the study was conducted by registering the post-operative MR image (in which the electrode placed within the deep brain is displayed) to the preoperative image using the non-rigid AtamaiWarp algorithm. The results showed that the displacement between the centre of the electrode after registration is within $0.73 \mathrm{~mm}$ of the planned surgical target on the pre-operative image, demonstrating negligible error caused by brain shift. 
Acknowledgements. The authors acknowledge the financial support from the Canadian Institute of Health Research (CIHR), the Ontario Research \& Development Challenge Fund (ORDCF), the Canada Foundation for Innovation (CFI), and the Ontario Innovation Trust (OIT).

\section{References}

1. C Hamani, E Richter, JM Schwalb, and AM Lozano, "Bilateral subthalamic nucleus stimulation for Parkinson's disease: a systematic review of the clinical literature," Neurosurgery, vol. 56, pp. 1313-1324, 2005.

2. EO Richter, T Hoque, W Halliday, AM Lozano, and JA Saint-Cyr, "Determining the position and size of the subthalamic nucleus based on magnetic resonance imaging results in patients with advanced Parkinson disease," J. Neurosurgery, vol. 100, pp. 541-546, 2004.

3. G Schaltenbrand and W Wahren, Atlas for Stereotaxy of the Human Brain. Stuttgart, Germany: Thieme, 1977.

4. KA Ganser, H Dickhaus, R Metzner, and CR Wirtz, "A deformable digital brain atlas system according to Talairach and Tournoux," Med. Imag. Analy., vol. 8, pp. 3-22, 2004.

5. KV Slavin, KR Thulborn, C Wess, and H Nersesyan, "Direct visualization of the human subthalamic nucleus with 3T MR imaging," Am. J. Neuroradiol., vol. 27(1), pp. 80-84, 2006.

6. SCL Deoni, BK Rutt, and TM Peters, "Segmentation of thalamic nuclei using high resolution quantitative magnetic resonance imaging and a modified k-means algorithm," in Proc. 13th ISMRM, no. 168, 2005.

7. KW Finnis, YP Starreveld, AG Parrent, AF Sadikot, and TM Peters, “Three-dimensional database of subcortical electrophysiology for image-guided stereotactic functional neurosurgery," IEEE Trans. Med. Imag., vol. 22(1), pp. 93-104, 2003.

8. PF D'Haese, E Cetinkaya, PE Konrad, C Kao, and BM Dawant, "Computer-aided placement of deep brain stimulators: from planning to intraoperative guidance," IEEE Trans. Med. Imag., vol. 24(11), pp. 1469-1478, 2005.

9. T Guo, KW Finnis, AG Parrent, and TM Peters, "Development and application of functional databases for planning deep-brain neurosurgical procedures," in Proc. MICCAI 2005, J Duncan and G Gerig (Eds.), LNCS 8749, pp. 835- 842, 2005.

10. E Cuny, D Guehl, P Burbaud, C Gross, V Dousset, and A Rougier, "Lack of agreement between direct magnetic resonance imaging and statistical determination of a subthalamic target: the role of electrophysiological guidance," J. Neurosurg., vol. 97, pp. 591-7, 2002.

11. C Hamani, EO Richter, Y Andrade-Souza, W Hutchison, JA Saint-Cyr, AM Lozano, "Correspondence of microelectrode mapping with magnetic resonance imaging for subthalamic nucleus procedures," Surg. Neurol., vol. 63(3), pp. 249-253, 2005.

12. YP Starreveld, "Fast nonlinear registration applied to stereotactic functional neurosurgery," Ph.D. dissertation, Univ. Western Ontario, London, ON, Canada, 2002.

13. T Guo, YP Starreveld, and TM Peters, "Evaluation and validation methods for intersubject non-rigid 3D image registration of the human brain," in Proc. SPIE Medical Imaging. Bellingham, WA: SPIE, 2005, vol. 5744, pp. 594-603, Visualization, Image-Guided Procedures, and Display.

14. KA Follett, MD Mann, "Effective stimulation distance for current from macroelectrodes," Exp. Neurol., vol. 92(1), pp. 75-91, 1986. 